\title{
Response: Brain miliary enhancement
}

\section{Joseph C. J. Bot ${ }^{1}$ (D) $\cdot$ Linda Mazzai ${ }^{1,2}$ (D) $\cdot$ Rogier E. Hagenbeek ${ }^{3}$ (D) Silvia Ingala ${ }^{1}$ (D) $\cdot$ Bob van Oosten $^{4}$ (D) Esther Sanchez-Aliaga ${ }^{1} \cdot$ Frederik Barkhof ${ }^{1,5}$ (D)}

Received: 29 February 2020 / Accepted: 10 March 2020 / Published online: 25 March 2020

(C) Springer-Verlag GmbH Germany, part of Springer Nature 2020

We thank Rice et al. for sharing their opinion with regard to the role and safety of brain biopsy in clinical diagnostic management of patients after reading of our paper.

In our paper, we describe multiple diseases that may present with miliary enhancement on brain MR images after intravenous contrast, some rare and some more frequently encountered. Within this summary, we also describe the variable

JCJB and LM are joint first authors.

This article is a reply to https://doi.org/10.1007/s00234-020-02381-4 and https://doi.org/10.1007/s00234-019-02335-5.

Joseph C. J. Bot

j.bot@amsterdamumc.nl

Linda Mazzai

1.mazzai@amsterdamumc.nl

Rogier E. Hagenbeek

r.hagenbeek@haaglandenmc.nl

Silvia Ingala

s.ingala@amsterdamumc.nl

Bob van Oosten

bw.vanoosten@amsterdamumc.nl

Esther Sanchez-Aliaga

e.sanchez@amsterdamumc.nl

Frederik Barkhof

f.barkhof@amsterdamumc.nl

1 Department of Radiology and Nuclear Medicine, Amsterdam UMC Location VUmc, P.O. Box 7057, 1007, MB

Amsterdam, The Netherlands

2 Institute of Radiology, Department of Medicine (DiMED), University of Padova, Padova, Italy

3 Haaglanden Medisch Centrum, The Hague, The Netherlands

4 Department of Neurology, Amsterdam UMC Location VUmc, Amsterdam, The Netherlands

5 Institutes of Neurology and Healthcare Engineering, UCL, London, UK efficacy of corticosteroid treatment on MR abnormalities as a possible consideration within a diagnostic paradigm and, as supported by previous papers, we describe the possible strong response of lymphoma to corticosteroids.

However we did not intend to describe nor want to advocate the strategy that, in case lymphoma is suspected in a patient, a trial of corticosteroid therapy should prevail before brain biopsy as we are very aware of the possible complications for histopathological examination as rightfully mentioned by Rice et al.

Within the text of our paper, we used in context of brain biopsy the term "last resort" which may dramatize the procedure of brain biopsy possibly too much, and we also agree that with current imaging and surgical techniques complication risk is acceptable when the high diagnostic yield is considered. However, we do want to stress that biopsy of the brain should only be considered when anamnesis in combination with other less invasive diagnostic procedures fail, especially in case of these very small biopsy targets.

Funding information No funding was received for this study

\section{Compliance with ethical standards}

Conflict of interest FB is supported by the NIHR Biomedical Research Centre at UCLH. All other authors have no conflict of interest.

Ethical approval All procedures performed in the studies involving human participants were in accordance with the ethical standards of the institutional and/or national research committee and with the 1964 Helsinki Declaration and its later amendments or comparable ethical standards.

Informed consent This was not a research study and, therefore, informed consent was not required.

Publisher's note Springer Nature remains neutral with regard to jurisdictional claims in published maps and institutional affiliations. 